EPJ Web of Conferences 52, 04008 (2013)

DOI: 10.1051/epjconf/20135204008

C) Owned by the authors, published by EDP Sciences, 2013

\title{
A Quark Matter Contribution to the Cosmic Ray Spectrum
}

\author{
Kyle Lawson ${ }^{1, a}$ \\ ${ }^{1}$ Department of Physics and Astronomy, University of British Columbia, Vancouver, BC, V6T 1Z1, Canada
}

\begin{abstract}
I will describe a possible dark matter model in which the dark matter is composed of heavy "nuggets" of standard model quarks and antiquarks bound in a high density phase of QCD. If objects of this type are formed early in the universe's history they may provide the observed dark matter content. In this scenario the nuggets are dark not because of their fundamentally weak interactions but because of the incredibly small number density required to explain the observed mass density of the dark matter. The correspondingly small flux of these objects through the earth renders them invisible to conventional high sensitivity dark matter searches intended to detect weakly interacting particles with a flux many orders of magnitude larger. Instead the greatest search potential for dark matter models of this form may come from the largest scale cosmic ray detectors. I will briefly describe the properties of quark nugget dark matter and then use these properties in order to predict the signal they would produce in a variety of cosmic ray detectors.
\end{abstract}

\section{Quark matter as dark matter}

Conventional dark matter models generally assume the existence of a new fundamental particle which interacts only weakly with the particles of the standard model and which carries a conserved charge to prevent its decay over cosmological timescales. The properties of this new particle are then to be extracted from the observed properties of the dark matter. As an alternative to invoking new fundamental physics there exists a class of dark matter models in which the dark matter is a composite object consisting of standard model particles. In the dark matter model considered here the dark matter takes the form of nuggets of standard model light quarks locked in a colour superconducting phase (or whatever phase is actually realized in the high density limit of QCD.) It has been speculated that such objects could be formed at QCD phase transition of the early universe at which time the essentially "freeze out" of interactions with the surrounding plasma. This model is similar to earlier objects such as "stranglets" in which the high density phase is stabilized by the presence of strange quarks [1].

The particular model considered here differs from earlier composite dark matter candidates in that these objects may be composed of either matter or antimatter and was originally proposes as an explanation of the matter dominance of the universe. It is assumed that the very early universe contained equal amounts of matter and antimatter and that a baryogenesis process must occur resulting in the present day matter dominance. In this scenario the dark matter forms through a charge separation mechanism in which the formation of nuggets of antimatter proceeds slightly faster than the rate of matter nugget formation. Af-

\footnotetext{
aklawson@phas.ubc.ca
}

ter the phase transition the hadronic matter not bound in nuggets annihilates with the remaining antimatter. However the remaining plasma has an excess of quarks corresponding to the excess of antiquarks locked in the nuggets. The remaining matter makes up the visible component of the universe. This mechanism can explain the observed visible matter to dark matter ratio if the matter component of the universe is composed of dark antimatter nuggets, dark matter nuggets and visible matter in a 3:2:1 ratio. More details on this model may be found in [2], [3], [4] and the references therein.

\section{Nugget structure}

At asymptotically large densities the ground state of QCD is a colour superconducting quark matter state with equal numbers of $\mathrm{u}, \mathrm{d}$ and s quarks. This state is electrically and composed purely of the three light quark flavours. However, at the surface of a nugget composed of quark matter the density must drop to zero from its bulk value. As the density drops so does the ability of the quark matter to support a strange quark population of the heavier strange quarks. As such the outer shell of the quark matter contains only $\mathrm{u}$ and d quarks and carries a strong electrical field due to the charge difference between these two species. At the nugget surface the energy density of these electric fields will be near the QCD energy scale. In order to maintain overall electrical neutrality the nugget acquires a surrounding shell of electromagnetically bound leptons known as the electrosphere (though in the case of an antiquark nugget the electrosphere is in fact composed of positrons.) The electrosphere was modeled in detail in [5] and runs from nuclear scale densities near the surface 
down to atomic densities far from the quark matter where the electrons see only a strongly screened central charge.

While the actual nugget structure may be rather complicated depending on the specific QCD phase realized many of these details are incidental to the general effects that I will consider below. Of primary importance is the presence of a higher than nuclear density quark matter core in a non-baryonic phase and a surrounding electrosphere of electrons or positrons.

\section{Observational constraints}

While, as argued above, the nuggets are effectively "dark" their interaction with visible matter that they do encounter is by not weak in any sense. It is only the rarity of these interactions makes them difficult to observe. At present the best constraints on the model are derived from astrophysical observations. Of particular interest are observations of regions where both the visible and dark matter densities are substantially above average. Here I briefly outline some of the relevant observations.

A stronger than expected $511 \mathrm{keV}$ line, associated with low momentum electron positron annihilation, is observed from the galactic centre [6]. This indicates the presence of significantly more antimatter in the galactic centre than is predicted by standard astrophysical models and may be explained by the annihilation of galactic electrons in the outer, low temperature, positron layers of a population of quark nuggets [7].

The COMPTEL satellite observes an excess of diffuse gamma rays from the galactic centre at the $\mathrm{MeV}$ energy scale. In the quark nugget model this emission is necessarily co-produced with the $511 \mathrm{keV}$ line [8]. While the majority of galactic electrons incident on a nugget will annihilate almost immediately on contact with the outer layers of the electrosphere a small fraction will be capable of penetrating sufficiently deep into the nugget that they annihilate with positrons carrying momenta near the binding energy scale of the quark matter. Detailed calculations of the structure of the positron layer and annihilation rates produce a $511 \mathrm{keV}$ to $\mathrm{MeV}$ emission ratio consistent with that observed [5].

The annihilation of galactic protons is a more complicated many body problem than the case of electrons. A proton colliding with the nugget will penetrate into the nugget and annihilate to jets of light mesons. This process results in two distinct annihilation signals. If one of the resulting jets is directed back towards the nugget surface the energy will rapidly heat a region of the positron layer which will subsequently emit in the xray band [9]. Jets unable to reach the surface dissipate their energy in the nugget increasing its temperature and resulting in thermal emission. The scale of the thermal spectrum is determined by the surrounding matter density and for the galactic centre will be in the microwave band [10]. In both of these bands an excess emission above expected galactic backgrounds are detected. The cHANDRA telescope detects an excess of emission from the galactic centre which it characterizes as consistent with an $8 \mathrm{keV}$ plasma though such a plasma is too hot to remain bound to the galaxy and has no obvious heating mechanism [11]. At the microwave scale the WMAP satellite observes a "haze" associated with the galaxy. The scale of both of these diffuse sources is consistent with the predictions of the quark nugget dark matter model.

The observations outlined above all arise from the interactions of visible and dark matter in the relatively high density region near the galactic centre. Recent work has also analyzed the contribution of these objects the cosmological background [12]. The density of the universe at very early phases of it's evolution was comparable to that of the galactic centre today and, as such, we would expect a similar diffuse radiation contribution to that from the galactic centre but redshifted down in energy by roughly three orders of magnitude. In fact, sky temperature measurements by the ARCADE2

While none of these sources independently provide a smoking gun the absence of any one of these sources, in roughly the observed ratio, would have immediately ruled out this dark matter model. Based on the observed scale of emission and assuming a typical dark matter profile for the galactic centre we may estimate the average quark nugget mass required to produce a diffuse continuum at this scale (smaller nuggets have a larger number density and thus produce a stronger diffuse emission.) A rough fit to the galactic backgrounds would suggest that the nuggets have an average baryonic charge of $B \sim 10^{25}$ or, equivalently, a mass of roughly a gram.

\section{Estimated flux and energy}

For present purposes the most important consequence of this model is the presence of massive nuggets of nuclear density antiquarks in the solar neighbourhood. Given a local dark matter density of roughly $1 \mathrm{GeV} \mathrm{cm}^{-3}$ and a galactic velocity of $200 \mathrm{~km} / \mathrm{s}$ the flux of quark nuggets is,

$$
\frac{d N}{d A d t}=n v \approx\left(\frac{10^{25}}{B}\right) k m^{-2} y r^{-1} .
$$

Where the nuggets' average baryon charge, B, should be taken to be around $10^{25}$. While this flux is far below the sensitivity level of conventional dark matter searches it is accessible to large scale cosmic ray detectors being comparable to the cosmic ray flux near the GzK limit.

A nugget of quarks will deposit thermal energy in the atmosphere as it collides with atmospheric molecules, however, this process will produce only modest levels of ionization and will not generate any relativistic secondary particles. As such the prospects for detecting these events in a cosmic ray experiment are very limited. If however the nugget is composed antiquarks the resulting annihilation of atmospheric molecules will release considerably more energy, much of it in the form high energy secondary particles. In order to estimate energy scale of these events we consider the case in which all of the atmospheric material in the path of the nugget annihilates completely. The atmospheric depth is on the order of $1 \mathrm{~kg} \mathrm{~cm}^{-2}$ and a quark nugget with a baryon number on the order of $10^{25}$ will 
have a radius of $10 \mu \mathrm{m}$ so that the energy generated by annihilations may be estimated as

$$
E_{\text {tot }}=2 X_{a t} c^{2} \pi R_{n}^{2} \approx 10^{26} \mathrm{eV}\left(\frac{R_{n}}{10^{-5} \mathrm{~cm}}\right)^{2} .
$$

While much of this energy will be thermalized within the nugget some will be released into the atmosphere and may be observable. Some discussion of the nuggets' thermodynamics and interactions with the atmosphere is required before this energy can be translated observational consequences. However the scale of the total energy produced as an antiquark nugget crosses the atmosphere is large enough to suggest that these events may be observable to detectors of sufficient area.

\section{Atmospheric fluorescence}

The production of atmospheric fluorescence requires a large number of relativistic secondary particles. As these particles lose energy to the surrounding atmosphere they excite nitrogen molecules which subsequently emit in the UV band. The fluorescence yield of a quark nugget induced air shower may thus be estimated from the number of high energy secondary particles which the nugget injects into the atmosphere.

Following the same logic which lead to equation (2) the total number of nucleons striking the nugget will be,

$$
E_{t o t}=\frac{X_{a t} \pi R_{n}^{2}}{m_{N}} \approx 10^{16} \mathrm{eV}\left(\frac{R_{n}}{10^{-5} \mathrm{~cm}}\right)^{2} .
$$

Where $m_{N}$ is the average mass per nucleon. This number however represents only an upper limit on the number of annihilations as thermal and radiation effects will likely cause some fraction of the incoming matter to be deflected before reaching the nugget's surface [13].

In order to estimate the number of secondary particles which these nuclear annihilations will produce consider the fate of an atmospheric nucleus incident on the surface of an antiquark nugget. Rather than annihilating directly on contact the nucleus will penetrate some distance into the nugget due to the relatively small wave function overlap between the nucleons and the cooper pair states of the quark matter. When the nucleon annihilates the annihilation products will stream off as hadronic jets along with the other light modes of the superconductor. As the nucleus' initial momentum is negligible in comparison to the total energy released the resulting jets will be randomly oriented relative to the nugget's surface. If one of these jets happens to be directed back towards the quark surface any components of the particle cascade which are weakly coupled to the quark matter may escape across the surface. Strongly bound hadronic particles will remain bound to the nugget while electrons and positrons are unlikely to be able to be able to penetrate out through the electrosphere and the associated strong electric fields. Consequently, muons produced in either the initial annihilation or the subsequent cascade will dominate particle emission from nugget (thought the total energy release is dominated by thermal photons.) The partial deflection of incoming atmospheric matter combined with the relatively small number of annihilation products may push the number of emitted secondary particles below the annihilation rate (3) by up to a few orders of magnitude however, even with these suppression effects, the total number of secondaries will remain at least comparable to the number generated in a ultra high energy cosmic ray induced air shower. Shower evolution is then dependent on the thermal properties of the nugget and the surrounding atmospheric density. The evolution of these parameters with atmospheric depth has been discussed in [13] where it is argued that the shower evolution may in fact take a more complex form then simply tracking the rise in atmospheric density.

The most important distinguishing feature of the air shower associated with a quark nugget passing through the atmosphere is the distinctive time scale involved. While the emitted secondary particles travel at essentially the speed of light the nugget itself moves at a much slower galactic speed. This results in an air shower which may evolve over tens of milliseconds.

\section{Surface particle counts}

In a conventional air shower the production of secondary particles continues only as long as the shower contains particles of sufficient energy to break up atmospheric nuclei. Beyond the point where ionization becomes the dominate interaction secondary particles cease to be created and only those particles with sufficient momenta will reach the earth's surface where they may be detected. Conversely, a quark nugget traveling through the atmosphere will continue to emit secondary particles across all atmospheric depths. These secondary particles are preferentially "beamed" in the forward direction of the nugget's path as the majority of annihilations will occur on the nugget's forward surface and only those annihilation components directed back towards the nugget surface are able to escape from the quark matter. The injection energy of these secondary particles depends only on nuclear physics processes within the nugget and consequently will be essentially constant across all atmospheric depths. Rather than producing the typical "pancake" of comoving secondary particles observed in a conventional shower the nugget will carry with it a cone of secondary particles emitted along its line of motion.

The nuggets themselves will move at a much slower velocity than the secondary particles it emits (unlike conventional showers where both the primary and secondary particles move at the speed of light.) This introduces a much longer timescale for the arrival of shower components. In [13] this timescale was estimated as follows. The highest energy shower components are likely to be muons with QCD scale energies $(E \sim G e V)$ in the reference frame of a surface observer these particles have a lifetime on the order of $10 \mu \mathrm{s}$ during which time they travel a couple kilometers. Thus the cone of relativistic muons will extend roughly a kilometer ahead of the nugget. Once this cone reaches the surface relativistic muons will begin 
to be seen in surface detectors. As the nuggets travel at velocities $v \sim 200 \mathrm{~km} / \mathrm{s}$ it will take on the order of a few milliseconds for the nugget itself to reach the surface at which point the emitted muons will be entirely absorbed by the earth and will no longer be detectable. A surface detector will thus detect particles over timescales from the millisecond down depending on the distance of the detector from the shower centre.

\section{Radio signal}

The secondary particles emitted by the nugget will be deflected by the earth's magnetic field leading to the emission of geosynchrotron radiation. This effect has also been considered in the case of air showers induced by a single ultrahigh energy primary and would allow for observations with a much higher duty cycle than conventional fluorescence detection.

As with the case of atmospheric fluorescence the total magnitude of the atmospheric radio signal will be comparable to that of a conventional air shower. This is for the simple reason that total number of contributing relativistic particles (which sets the magnitude of both the fluorescence and geosynchrotron signals.) As with the fluorescence signal the clearest differentiating feature between a quark nugget driven air shower and that of a ultra high energy cosmic ray is in the signal timing. The fact that the nugget travels considerably slower than the speed of light causes the radio emission to occur over millisecond time scales.

A second significant distinguishing feature of a quark nugget air shower in the radio band arises from the fact that the nugget itself is heated as it passes through the atmosphere reaching keV scale temperatures near the earth's surface. Despite the small area of the emitting surface such a high temperature can result in appreciable levels of thermal radiation. While this radiation is generally an order of magnitude smaller than the associated geosynchrotron signal it is spread across a broader range of wavelengths and a wider range of emission directions [14].

As the nugget's temperature is set by the density of the surrounding material the thermal emission may be boosted once the nugget enters the earth. It has been suggested [15] that the thermal emission from a nugget passing through the radio transparent antarctic ice will present a relatively strong signal across the radio band.

\section{Conclusion}

If the dark matter in our galaxy consists of nuggets of quark and antiquark matter then these nuggets, while evading conventional dark matter searches, may be detectable by large scale cosmic ray detectors. If a nugget of antimatter strikes the earth's atmosphere it will induce an air shower of secondary particles at a scale similar to that produced by a single ultra high energy cosmic ray. These air showers are detectable by the same means currently used to detect air showers associated with ultrahigh energy cosmic rays. The main distinguishing feature of this type of shower is the significantly longer time duration associated with the relatively low velocity of the primary. If currently operating or planned cosmic ray detectors are capable of searching for signals on this longer (millisecond) timescale they will be able to explore most of the interesting parameter space for dark matter of this form.

\section{References}

[1] Witten, E., Phys. Rev. D 30, (1984) 272-285

[2] Zhitnitsky, A., JCAP 10, (2003)

[3] Oaknin, D. and Zhitnitsky, A., Phys. Rev. D 71, (2005) 023519

[4] Zhitnitsky, A., Phys. Rev. D 74, (2006), 043515

[5] Forbes, M.M., Lawson, K. and Zhitnitsky, A., Phys. Rev. D 82, (2010), 083510

[6] Knodlseder, J. et al., Astron. and Astrophys. 441, (2005) 513-532

[7] Zhitnitsky, A., Phys. Rev. D 76, (2007) 103518

[8] Lawson, K. and Zhitnisky, A., JCAP 0801, (2008), 22

[9] Forbes, M.M. and Zhitnitsky, A., JCAP 0801, (2008), 23

[10] Forbes, M.M. and Zhitnitsky, A., Phys. Rev. D 78, (2008), 083505

[11] Muno, M.P. et al, Astrophys. J. 613, (2004), 326-342

[12] Lawson, K. and Zhitnitsky, A.R., arXiv:1210.2400

[13] Lawson, K., Phys. Rev. D 83, (2011), 103520

[14] Lawson, K., arXiv:1208.0042

[15] Gorham, P., Phys. Rev. D 86, (2012), 123005 